\title{
XVIII.
}

Aus der k. k. chirurgischen Klinik in Kraka u zur Zeit der

Direction des Prof. Rydygier.

\section{Ueber Lipome des Samenstranges}

\author{
Von \\ Dr. Anton Gabryszewski, \\ g. I. Assistent an der chirurgischen Klinik.
}

Vor einigen Jabren kam in der chirurgischen Klinik ein Fall vor, der, als "rara avis", schon durcb die Schwierigkeit der Diagnose längere Zeit uns beschäftigte, - und der vorher Ursache unangenehmer therapeutischer Täuschungen seitens erfahrener Aerzte war. Aus praktischen Rücksichten beschloss ich, diesen Fall zu veröffentlichen.

Die Zusammenstellung analoger Fälle aus der Literatur machte solche Schwierigkeiten, dass ich schon die undankbare Arbeit aufgeben wollte, als die Veröffentlichung der Pariser These von Saraziu mich von Neuem zul Ausführung des früheren Vorhabens ermuthigte

Als Einleitung zu dieser Arbeit führe ich zuerst die Beschreibung unseres Falles an, und zwar in der Form, in welcher ich sie vor einigen Jahren niedersclurieb:

Im October 1892 meldete sich in der Krakaner Kilinik Herr J. C., ein ziemlich alter Mann, der eine grosse Hodensackgeschwulst hatte und über einige Aerzte sich beschwerte, die ihm das Wasser aus der Geschwulst herauszulassen versprochen hätten; aber trotz wiederholter Punction mit dem Troicart nichts zu 'Tage förderten; vielmehr fing der IIoden an, sehr schmerzhaft zu werden. - Dies veranlasste ihn, Rath in der Klinik zu suchen.

Da die Geschwulst eine Hydrocele zu sein schien, so musste man die Lntersuchung desto genauer durchführen. Aus der Ansmnese erfuhren wir, dass die Eltern des Kranken im hohen Alter an Lungenentziundung starben und vorher nie krank gewesen sind; zwei Brider und drei Schwestern erfreuen sich trotz hohen Alters der besten Gesundlieit. Der Kranke selbst hatte früher einen weichen Schanker und einen Tripper gehabt; beides 
ist aber bald und ohne Anstand ausgeheilt. Syphilis hat er nicht durchgemacht. In Wien wurden ihm Nasenpolypen operirt, auch litt er vor einigen Jahren an Unterschenkelgeschwuren, welche lange und hartnäckig nicht heilen wollten, bis im Krankenhause eine schwere Form von Erysipel sich hinzugesellte, die ihn beinahe ums Leben gebracht hätte; nach Ábheilung der Rose aber verschwanden die Geschwüre und recidivirten nicht mehr.

Das gegenwärtige Leiden nahm seinen Anfang vor ungefăhr zwei Jahren ohne irgend welche Veranlassung. Eines Tages bei einer Besichtigung des Hodensacks fühlte der Kranke in diesem Organ eine kleine Geschwulst, die seine Aufmerksamkeit auf sich lenkte. $O b$ das ein geschwollener Hoden war, oder ob etwas neben dem Hoden zu wachsen begann, kann er nicht ganau angeben. Dagegen behauptet er mit Sicherheit, vorher keine Spur von Bruch bemerkt zu haben. Anfangs beachtete er die Geschwulst gar nicht, zumal da sie ihm weder Beschwerden, noch Schmerzen verursachte. Als sie jedoch schnell zu wachsen und ihm beim Gehen hinderlich zu sein anfing, sah er sich veranlasst, ärztliche Iülfe aufzusuchen.

Wie schon erwähnt, erklärte der Arzt, dass in der Geschwulst eine Flüssigkeit sei, die herausgelassen werden müsse. Als aber der Kranke, nach ziemlich langem Zögern, auf diesen Eingriff einwilligte, und die gemachte Punction einen positiven Erfolg nicht ergab, begab er sich in die Krakauer chirurgische Universitätsklinik, wo er am 17. October aufgenommen wurde.

Die Untersuchung des Kranken ergab folgendes: Patient ist 57 Jahre alt, von mittlerer Grösse, stark gebaut, in gutem Ernährungszustande. Die inneren Organe sind bis auf ein unbeträchtliches Lungenemphysem ohne krankhafte Veränderungen. Das Fettpolster ist gleichmässig vertheilt, aber etwas stark. Der Hodensack ist durch eine rechtsseitige, kindskopfgrosse, eiförmige Geschwulst vergrössert. Diese Geschwulst reicht bis an das $P$ oupart'sche Band, ja sie scheint sogar bis an die äussere Oeffnung des Leistenkanals sich zu erstrecken, ragt jedoch nicht in den Kanal selbst hinein. Die Oberfläche ist glatt und zeigt eine ungewöhnlich deutliche Fluctuation nach allen Richtungen hin. Die Haut des Hodensacks ist mässig gespannt, mit vielen Venen bedeckt; der Penis ist voch sichtbar, obwohl zum grössten Theil in der Haut, die die Geschwulst überzieht, versteckt. Der Hoden ist nicht durchzufühlen, lässt sich aber im unteren, hinteren und inneren Segment der Geschwulst nachweisen, da ein Druck auf diese Stelle das dafïr charakteristische Schmerzgefühl hervorruft. Ein Durchscheinen ist nicht vorhanden. Der Perenssionschall ist uberall gedämpft. Beiro Pressen und Hasten vergrössert sich die Geschwulst nicht; aus dem Leistenkanal schiebt sich nichts heraus, ein Kollern ist nicht wahrnelımbar.

Auf Grund dieses Befundes konnte man eine durch Blutaustritt getrübte Hydrocele diagnosticiren oder auch eine Hämatocele, ferner eine Laparoceie oder endlich eine Chylocele oder Galactocele, (weniger eine Spermatocele). Um dies festzustellen, musste man noch eine Probepunction machen. Dies thaten wir auch mehrfach mit einer ziemlich dicken Nadel an mehreren Stellen; das Ergebniss war aber vegativ, denn nicht einmal eine Spur von Flüssigkeit war zu finden. 
Dies bestärkte uns darin, dass wir es mit einer festen, aber so weichen Geschwulst zu thun haben, dass sie ausgeprägte Merkmale von Fluctuation zeigt. Beim genauen und starken Abtasten uberzeugten wir uns, dass diese Geschwulst in ihren centralen Theilen hart war und den Eindruck machte, als ob man einen festen Körper umtastete, der von einer Flüssigkeit umgeben ist. Derartige Neubildungen könnten sein: Hin Myxoma, If yxosarcoma und Lipoma - alles selır seltene Tumoren, wie wir es im K och er'schen Werke: „Leber die Krankheiten der männlichen Geschlechtstheile", gefunden haben.

Eine exacte Diagnose war also nicht möglich, jedoch war für mich die Diagnose: Lipoma funiculi spermatici, die walurscheinlichste.

Mit gatiger lirlaubniss meines hochverehrten Chefs Herrn 'rof. Rydygier, unternahm ich am 20. Octuber in der Narkose die Operation. Ich machte einen Lüngsschnitt vom I'o u pa r' t'schen Bande nach unten, ungefähr $15 \mathrm{Cm}$. lang; nuch Durchschneidung der Haut und der dünnen Tunica Dartos trat dic Geschwulst als ein walzenförmiger, glatter, mit einer harten, glänzenden Hall: bedeckter Körper hervor. Nach Spaltung dieser Haut zeigten sich sofort im Schnitt die das Lipom kennzeichnenden Fettklumpen und begannen, sich nach aussen hervorzustülpen. Das Iferausnehmen der Geschwnlst aus der Höhle gelang mit Leichtigkeit und schnell; das Lipom war nur mit den Venen des Plexus pampiniformis eng verwachsen und erforderte beim Trennen einige Sorgfalt. 'Trotz grosser Vorsicht und Aufmerksamkeit trat dennoch eine unbedentende Naclublutung ein, die eine Art von Hämatom zur Folge hatte und so eine schuelle Ileilung verzögerte. Das Vas deferens und die Arterien liessen sich mit Leichtigkeit isoliren, der Hoden war weder ver-, noch durchwachsen; dio äussere Oeffnung des Leistenkanals war durch die in ihn hineinragende Spitze der Geschwulst etwas erweitert.

Hierauf nähte man sowohl die Tunica vaginalis communis, wio die Haut vollständig zu und versah die Wunde mit einem gewöhnlichen aseptischen Verbande.

Del Verlauf post operationem war fieberfrei.

Nach 8 Tagen Verbandwechsel, Heilung per primam, nur die rechte Hälfte des Ilodensacks war infolge von Nachblutung, deren Spuren in den schwarzen und blauen Sugillationen auf der llaut des IIodensacks sichtbar waren, vergrössert. leh öfhete mit einer gewöhnlichen Kornzange die Höhle und liess ein wenig Blut und Coagula heraus; trotzdem verschwand die Anschwellung nicht vollstindig. Auch als der Kranke nach 3 Wochen die Klinik verliess, war sie noch vorhandes.

Erst nach einem Jahre, unter der Linwirkung von warmen Bädern und einel Jodsalbe, kehrten mit dèr Zeit fast vollstindig normale Verhälnisse wieder zurïck nnd, wie ich es feststellen konnte, war weder ein Revidiv, noch eine Atrophie des llodens eingetreten.

Die herausgenommene Geschwulst stellte ein ziemlich grosses Lipom dar, von lappenförmiger Oberfläche, das schwach mit Blutgefässen versehen und weich war. Es wog tast 5 l'fund. Auf dem Durchischitt sah man, dass der centrale 'lheil viel härter war und sich durch eine mehr graue Farbe vom Reste der Geschwulst minerschied. Von diesem in der Mitte gelegenen Theile gingen strahlenförmig peripherwärts nach allen Riuhtungen 
hin bindegewebige Streifen, die dann immer dünner und seltener wurden. Mikroskopisch wurde die Diagnose: ein grosszelliges, gelapptes Lipom, das central mit Bindegewebszügen stark durchwachsen war (Fibrolipoma „tumeur adipofibreuse"), bestätigt.

Dieses Lipom ging unzweifelbaft aus dem Fette bervor, das gewöhnlich in dem dünnen Gewebe liegt, das um die Blutgefässe, das Vas deferens und den Samenstrang, innerhalb der Tunica vaginalis communis sich befindet, und, umgeben von einer verdickten Haut dieser gemeinsamen Scheide, gab es Zeichen einer wirklichen Fluctuation, welche die Untersuchung und die Diagnose ersehwerte.

Dieser vielfach merkwürdige Fall, von welchem die specielle Krankengeschichte eine genaue Vorstellung giebt, schien etwas sehr Seltenes zu sein, da Kocher in seinem umfangreichen Werke: ${ }_{n}$ Ueber die Krankheiten der männlichen Geschlechtsorgane", davon kaum einige Fälle anzuführen vermochte.')

Als ich überdies wichtigere chirurgische Werke, sowohl Handbücher wie Monographien, sorgfältig durchsuchte, bemerkte ich, dass sie alle diesen Gegenstand ungenau oder geradezu falsch behandeln. Sie sprechen über diese Neubildung wie über eine gewöhnliche Sache und fubren keine wichtigeren Beispiele aus der Literatur an oder berufen sich ohne weiteres auf einige bekannte Fälle. ${ }^{2}$ )

Als es mir daher gelungen war, eine ganze Reihe hier und da zerstreuter Fälle, von denen die Autoren meistentheils gegenseitig Nichts zu wissen scheinen, zu sammeln, hielt ich es für angezeigt, das gesammelte Material zusammenzustellen und kritisch zu behandeln, Unklares aufzuklären und Unsicheres festzustellen.

Als Lipome des Samenstranges bezeichnen wir Neubil dungen, welche aus dem Fettgewebe des Samenstranges oder auch aus dem subserösen Fettgewebe hervorgegangen sind, sich aber innerhalb der Tunica vaginalis communis entwickelt baben. Diese Definition ist sehr scharf, und man muss derartige Neubildungen entsehieden von ähnlichen pathologischen Vorgängen dieser Gegend unterscheiden, welche dort öfters sich vorfinden.

Die Lipome des Samenstranges, sind ein seltenes Leiden, und, wie schon erwähnt, haben wir uber sie nur wenige genauere $\mathrm{Be}$ richte, und auch diese erschienen erst vor nicht langer Zeit.

1) In dem trefflichen Werke Kocher's, „Ueber die Krankheiten der männlichen Geschlechtstheile“, ist der Abschnitt über die Lipome des Samenstranges sehr stiefmütterlich behandelt; das Ganze ist weniger eingebend dargestellt; die sich darauf beziehende Literatur ist nicht vollständig berücksichtigt.

2) In der uns am meisten zugănglichen deutschen Literatur giebt es überbaupt keine Monographie, nicht einmal eine Abbandlung aus diesem Gebiete. 
Zwar beschreibt schon Cloquet, der Schöpfer der interessanten Theorie von der Entstehung der Briiche'), im Jahre 1819 einen unzweifelbaften Fall von einem Lipom des Samenstranges, doch verdanken wir erst $\mathrm{Curling}$ eine genaue und ausfübrliche Beschreibung dieses Leidens, aus dem Jahre 1857. Nelaton behauptet in seinem Buche ther Chirurgie, dass Lipome des Samenstranges die häufigsten Neubildungen sind, die sich hier zeigen; einen Beleg dafiir fubrt er aber nicht an. Holmes, Annandale, Gascoyen, Deguise, Rossander, Gordon, Buck, Eben Watson geben meist kurze Bescbreibungen und bringen nichts Neues. - Wichtiger ist erst die Arbeit von Brossard (1884), der in den "Arcbives de médecine" einen Fall von einem grossen Lipom des Samenstranges genau bescbrieb und mit werthvollen Bemerkungen versah. Bald darauf veröffentlicbte $\mathrm{Br} 0$ ca einige - hauptsächlich an Leichen - beobacbtete Fälle und nach ihm Koch, Gross, Péan u. A. Virchow beribrt in seinem grossartigen Werke uber die Neubildungen nur oberflächlich die Lipome des Samenstranges, und Kocber schreibt, - wie schon gesagt - in der „Deutschen Chirurgie" über diese sehr flücbtig und berücksichtigt sehr wenig die Literatur. Ebenso wenig beschäftigt sich damit Reclus in seinem "Neuen Vortrage der Chirurgie". Den besten und am meisten erschöpfenden Aufsatz uber die Lipome des Samenstranges lieferte Gaston Sarazin in seiner "Thèse pour le doctorat en médecine", Paris 1895, und aus dieser Arbeit schöpfte ich den grössten Theil der später erwähnten Fälle, die ich in kurzen Umrissen anfubre.

Fall 1. Lipom des Samenstranges, gefunden an der Leiche eines Greises, Cloquet 1819. Die Geschwulst war einem nicht reponirbaren Netzbruch ähnlich (Epiplocele), der durch den Leistenkanal bis zum Bauchfell reichte.

Fall 2. Curling 1857. Lipom des Samenstranges. Die Geschwulst in der linken Hälfte des Hodensacks, die Anfangs von solchen Chirurgen, wie M. Thompson, Lawrence und Brodie für einen Netzbruch gehalten wurde, war mannskoptgross. La wrence machte im Jahre 1845 eine Operation - da er den Samenstrang nicht isoliren konnte, so exstirpirte er den Hoden. Die Geschwulst kehrte trotzdem wieder, und Curling

1) Cloquet, ,Recherches sur les causes et l'anatomie des hernies abdominales." Thèse de Paris 1819.

Eigentlich spricht schon im Jahre 1750 Pelletan in dem 3. Bande seiner ,Cliniques" vom Lipom des Samenstranges wie von einer „hernie graisseuse"; Antangs meinte er. es sei eine Epiplocele, aber er fand keine Spur von Bruchsack. Eine Versanımlung der gelehrten Mitglieder der „Königlichen chirurgischen Akademie" schenkte diesem Berichte keinen Glaben, und die Sache wurde vergessen (Sarazin). 
schnitt sie zum zweiten Male heraus im Jahre 1849; 3. Recidiv und die 3. Operation im Jahre 1855.

Fall 3. Deguise. Ein Riesenlipom des Hodensacks. Ausschälung im Jahre 1859. Der Verfasser beschreibt eine mannskopfgrosse Geschwulst, die ans dem Hodensack eines jungen Soldaten ausgesehnitten wurde. Bei dieser Gelegenheit erzählt $\mathrm{Ch}$ assaign ac von einer ähnlichen Geschwulst bei einem Greise.

Fall 4. M. A ubert und I a rt. Lipom des Samenstranges, demonstrirt im ärztlichen Verein zu London (der Kranke ist gestorben) 1874.

Fall 5. Gordon-Buck. Lipom des Samenstranges von der Grösse einer Birne auf der rechten Seite. Enucleation, Tod 1871.

Fall 6. Rossander. Ein beiderseitiges Lipom des Samenstranges, das bis zum Bauchfell reichte und in das subperitoneale Fett überging.

Fall 7. Eben Watson. Fibrolipoma des rechten Samenstranges, von der Grösse einer grossen Birne, lange wie ein Bruch behandelt, der Anfangs klein und reponirbar war. Im Laufe von 9 Jahren hat es sich vergrössert und konnte zuletzt nicht reponirt werden; operirt mit gutem Erfolg.

Fall 8. Brossard. Lipom des Samenstranges, im Jahre 1882 beschreibt B. einen bei Cruveilhier beobachteten Fall. Bei einem 37 Jahre alten Manne bildete sich vor 6 Jahren eine kleine Geschwulst am linken Hoden, die dann mannskopfgross wurde und tief in den Leistenkanal hineinragte. Die Aerzte in der Heimat des Patienten machten eine Punction, nach welcher ein Eiterherd entstand. Letzterer wurde durch einen anderen Arzt punctirt, wonach viel Eiter ausfloss, und dann eine Fistel zurückblieb. Die Gesehwulst wurde zugleich mit dem an der medialen Seite gelegenen Hoden mit Loichtigkeit ausgeschält ; lappiger Bau, der Leistenkanal erweitert durch einen Ausläufer aus der Spitze der Geschwulst; unten ein orangengrosser Eiterherd, oben eine Höhle mit cystenartigem Inbalt. Die Geschwulst wog $4 \frac{1}{2} 2 \mathrm{Kgrm}$. Die Hautadern des Hodensacks waren stark erweitert.

Fall 9. R. M. Hodges. Ein seltener Fall von einem Lipom im Hodensack, das infoige von Fluctuation eine Cystocele zu sein schien. 1884, Boston.

Fall 10. Willemer sagt in seinem Berichte über den Fall von Hodges im Centralblatt für Chirurgie 1885, dass in Göttingen eine mannskopfgrosse Geschwulst operirt wurde, die man als ein Sarkom des Hodens diagnosticirte, und die sich dann als ein Lipcm des Samenstranges erwies. Der Hoden liess sich isoliren und wurde erhalten.

Fall 11. R. Park (Buffalo). Lipom des Hodens. (Zweimaliger Bericht in dem Centralblatt für Chirurgie im Jahre 1886 und 1888). Bei einem 43 Jahre alten Manne gab eine sehr grosse Geschwulst im Hodensack, die unter der Haut leicht beweglich war, Zeichen der Fluctuation; schmerzhaft war die Geschwulst nur da, wo der Hoden lag. Da die Gefässe des Hodens und des Samenstranges in die Geschwulst hineinragten, musste man bei der Enucleation der Geschwulst die Castration ausführen. Die Geschwulst wog 3 Pfund. Der Verfasser führt 3 Fälle von Kimball, Jobert und Deguise an.

Fall 12. J. Hutchinson. Lipome des Samenstranges. Der Ver- 
fasser erkannte ein grosses Lipom des linken Samenstranges und schälte es aus, ohne den Hoden zu verletzen. Er behauptet, dass die Diagnose schwer ist, nur Cooper habe sich nie geirrt.

Fall 13. Broca 1888. Lipom des Samenstranges, das in den Leistenkanal hineinragte - bei der Section festgestellt.

Fall 14. Broca. Lipom des Samenstranges auf der linken Seite, schiefer Bruch auf der rechten. Bei der Section stellte man eine gänseeigrosse Geschwulst fest, die fluctuirte und den Eindruck einer Hydrocele, oder eines Netzbruches (Epiplocele) machte. Es stellte sich heraus, dass es ein Lipom war, das bis in den Leistenkanal hineinragte, das Banchfell jedoch nicht, berührte.

Fall von Broca. Leistenlipome älnlich einem Netzbruch. Multiple Lipome. Bei einem Kranken mit einem Lipom auf dem Halse un'd unter dem Kinn eine längliche Geschwulst im Leistenkanal, die B. ohne Operation und ohne Section als ein Lipom erkannte.

Fall 15. Broca. Lipom des Samenstranges, das den Hodenbruch umgab und gleichsam von allen Seiten umhlilte.

Fall 16. Broca. Lipome in der Leistengegend auf beiden Seiten. Auf der linken Seite umgiebt das Lipom den Bruch und ragt zugleich mit dem Samenstrange in den Leistenkanal binein; anf der rechten Seite liegt das Lipom zwischen der Tunica vaginalis communis und dem $M$. cremaster.

F a ll 17. De lbet. Lipom des Samenstranges, das in den Leistenkanal hineinragte, bis zum Bauchfell reichte und mit diesem verbunden war, 1888.

Fall 18. Gross beschreibt ein Lipom des Samenstranges, welches $1200 \mathrm{Grm}$. wog, und das er einem 70 jahrigen Greise ausschälte. Der Hoden war ganz frei; Ausgangspunkt - subperitoneales Fettgewebe, 1890.

Fall 19. Koch. Lipom des Hodensacks 1890. Bei einem 49 Jahre alten Manne, der nie einen Bruch gehabt hatte, entwickelte sich die Geschwulst allmählich, reichte bis zu den Kuien, war elastisch und weich. Oben ging sie bis zu der äusseren Oeffnung des Leistenkanals. K. schälte die Geschwulst zugleich mit dem Hoden heraus (der abgesondert unten an der Geschwulst lag), da die Gefässe und der Samenkanal mitten durch die Neubildung liefen. Ausserdem holte er eine kleine Geschwulst aus dem Leistenkanal heraus, die vor der Operation ein Bruch zu sein schien.

Die Geschwulst wog 5 Pfund, war mit einer dicken, weissen, fibrösen Kapsel, wie auch mit einem serösen Ceberzug umgeben, der ganz in sich geschlossen war. Das Vorhandensein dieses Ueberzugs erklärt der Verfasser nicht als den Ueberrest eines früheren Bruchsacks, sondern als einen nicht ganz geschlossenen Processus vaginalis peritonei, der also nicht vollständjg verschwunden ist und sich in das Ligamentum vaginale nicht verwandelt hat. -

Der Verlauf post operationem war normal.

Fall 20. Reclus. Einem 62 jährigen Manne wurde ein Lipom des Samenstranges, das $59 \mathrm{Cm}$. Umfang hatte und $1600 \mathrm{Grm}$. wog, exstirpirt ohne dabei den Hoden zu verletzen. Den Ausläufer der Neubildung, der in den Leistenkanal hineinragte, zog man leicht mit einigen Zügen beraus.

Fall 21. Franeois Hue. Lipom des Samenstranges. Bei einem 64 Jahre alten Marne, der vor 19 Jahren rittlings auf einen Balken fiel, begann im Hodensack anf der rechten Seite eine Geschwulst sich zn bilden. 
Die Neubildung hatte $76 \mathrm{Cm}$. im Umfang, war stellenweise weich, stellenweise hart und verlängerte sich in den Leistenkanal. Man diagnosticirte ein Sarcom, da der Kranke kachektisch aussah. Die Operation, Exstirpation der Geschwulst und des Hodens, war leicht. Die Geschwulst wog 11 Pfund, war an dem oberen Ende ein reines Lipom, an dem unteren colloid entartet. (Rappoit par M. Paul Reynier).

Fall 22. T. Kocher 1887. Lipome des Samenstranges und der Scheidenhülle (Deutsche Chirurgie).

Der Verfasser giebt an, dass im anatomisch pathologischen Museum 2u Zürich ein Präparat sich befindet, das einen Samenstrang darstellt, der mit lappenförmigem Fettgewebe ausgefüllt ist, wie eine Wurst, die in den Leistenkanal bis zar inneren Oeffnung hineinragt. Das Lipom lässt sich leicht von den Blatgefässen, dem Samenkanal und der Scheidenbülle trennen.

Fall 23. Gascoyen exstirpirte ein Hodenlipom, das uber 5 Pfund wog (ebenso Lane) (K o c her).

Fall 24. Wilson operirte eine mit Geschwüren bedeckte Lipomgeschwulst des Samenstranges, die gegen 20 Pfund wog (Kocher).

Fall 25. Virchow führt an, dass im Berliner Museum ein grosses, $19 \frac{1}{2}$ Pfund wiegendes Lipom des Samenstranges sich befindet, das W ilms im Jahre 1854 herausnahm.

Fall Duplouy 1891. Die Neubildung gehörte dem linken Samenstrange an, war mannskopfgross und bestand aus 2 Theilen: die kleinere Geschwulst war ein Myxosarcoma, die grössere ein Lipom. Dieser Fall ist ein complicirter, es handelt sich um eine gemischte Neubildung, weshalb ich ihn auch in der Reihe vicht anführe.

Fall 26. Schwartz und Sarazin (Paris). Sarcom des Hodens und Lipom des Samenstranges. Ein 40 jähriger Mann bemerkte seit 2 Jahren eine Vergrösserung des Hodens ; Schmerzhaftigkeit und allgemeine Abzehrung.

Enucleation der fanstgrossen Geschwulst; es zeigte sich, dass ausser dem Sarcom des Hodens noeh ein Lipom im Samenstrang sich befand.

Fall 27. W. Anderson beschreibt ein beiderseitiges Lipon der Samenstränge, das als beiderseitiger Netzbruch (Epiplocele) diagnosticirt und von ihm operativ beseitigt wurde. Recidiv.

Nicht ganz sichere Fälle von Lipomen des Samenstranges, die K a r e w sk i, Holmes, Broca, Duplouy, Langenbeck, Tizzoni, Mackilvain, Lavrence u. A. beschrieben haben, beziehen sich entweder auf gemischte Neubildungen oder auf Geschwälste, die man für Lipome hielt, bei denen jedoch weder die Operation, noch die Section die Liagnose bestätigte.

Fall 28. Curling (s. Fall 2.) (Mal du testicule, traduit parGo s s eli $u$ ). Im Museum des chirurgischen Collegiums zu London befindet sich ein Präparat von einem Lipom des Samenstranges, das lose mit den Gefässen zusammenhängt, $12 \mathrm{Cm}$. lang und eiförmig ist. Die Neubildung zeigt einen lappenförmigen Bau.

F'all 29. A'lbert schreibt in seiner "Chirurgie", dass er ein faustgrosses Lipom sah, das die eine Hälfte des Hodensacks einnahm, durch den I_eistenkanal bis zum Bauchfell reichte und dort im subserösen Gewebe sich verbreitete.

F'all 30. Mein Fall, den ich Kingangs beschrieben habe. 


\section{Topographische und pathologische Anatomie - Pathogenese.}

Um die Patbogenese und die pathologisebe Anatomie dieses Leidens zu beschreiben, muss man, wenn auch in kurzen Ztigen, die anatomischen Verbältnisse in der Gegend des Hodensacks, des Samenstranges und des Leistenkanals sich vergegenwärtigen.

Wenn die Bruchoperation einen der interessantesten Theile der Akiurgie bildet und bei jedem neuen Fall neues Interesse stets erwecken wird, so verdankt sie dies in erster Linie dem verwickelten Bau dieser Gegend. „Es ist das Uralte und ewig Neue" sagt Hy rtl in seiner topographischen Anatomie. - Betrachten wir also die Schichten des Hodensacks. - Die Haut des Bauches gebt ununterbrochen auf die Hoden uber, wo sie dünner und faltig wird. Das subcutane Fettgewebe des Bauches verwandelt sich in ein dunnes subcutanes Gewebe, in dem niemals Fett ist, und in die Tunica dartos des Hodensacks, die mit glatten Muskeln reichlich versehen ist, und die eigentlich der Bauchhaut entspricht. Wenn wir weiter geben, finden wir diunnes Bindegewebe, das den Samenstrang um. hüllt, die sogenannte Tunica Cooperi (eigentlich Tunica Scarpae), die die Verlängerung der Fascie des oberflächlichen Hautmuskels des Bauches ist (eigentlich die sogenanuten Fibrae collaterales Winslowii). Man darf nicht vergessen, dass die zarte „Tunica Cooperi“ bei Leuten mit altem Bruch sich in eine dicke, starke Haut verwandeln kann. (ieht man weiter, so treffen wir schon den eigentlichen Samenstrang (Funiculus spermaticus), dessen oberste Schicht der M. cremaster bildet, ein Muskelbündel des inneren schiefen Bauchmuskels, der das Heben und Herablassen des Hodens zur Aufgabe hat. Unter diesem Muskel erst liegt die eigentliche Tunica vaginalis communis testis et funiculi spermatici oder Fascia infundibuliformis, die die Verlängerung der Fascia transversa ist und alle Bestandtheile des Stranges umbiillt. Diese sind der Reibe nach aufgezäblt: 1. Vas deferens, 2. Arteria spermatica, :3. zwei Venengeflechte, der sogenannte Plexus pawpiniformis, t. ein sympathischer Nerv, der sogenannte Plexus spermaticus nervi sympathici, 5. Ueberreste des Processus vaginalis peritonei, welcher die Tunica vaginalis testis propria bildet und in Samenstrange als ein sebr dïnnes Band verläuft, das sogenannte Ligamentum filiforme von Krause, Habenula Halleri, Ruinae processus vaginalis. Ferner sind noch zu erwähnen: Art. sperm. ext. s. cremastica, A. deferentialis, analoge Venen und der Nervus sperm. ext. (Hyrtl-Tillaux). Die eigentlichen Theile des samenstranges sind durch dunnes Bindegewebe mit einander verbunden, in 
dem gewöhnlich kleine Fettläppchen liegen. Diese sind in den allermeisten Fällen der Ausgangspunkt der Nenbildung. Im Leistenkanal selbst verliert der Samenstrang allmählich seine tieferen Schichten und Bestandtheile; es bleibt also in der Apertura ext. canalis ing. die Tunica Cooperi; in den M. obliquus abd. int. gebt der M. cremaster uber, und die Tunica vaginalis communis verliert sich in der Fascia transversa. Hier gehen jetzt, wie aus einer plötzlich geöffneten Scheide, Gefässe, Nerven und das Vas deferens auseinander; diesen folgt das dunne Bindegewebe mit seinen Fettläppchen und den Ueberresten des Processus vaginalis und vereinigt sich oder, besser gesagt, geht in das subseröse Gewebe, das mit Fett reichlich ausgepolstert ist, uber. Dies ist die innere Oeffnung des Leistenkanals und das Ende der Verbindung des Hodens mit dem Bauchfell. Wie wir uns bald überzeugen werden, gehen auch von diesem Fettgewebe ziemlich häufig Lipome des Samenstranges aus.

\section{Woher nehmen Lipome des Samenstranges ihren Anfang?}

Die Beantwortung dieser Frage ist weder leicht, noch sicher. Virchow zählt diese Geschwülste zu den sogenannten Lipomata capsularia, die sich in einem Organ entwickeln, und nennt sie Lipoma herniosum. Er selbst giebt aber zu, dass versehiedene Autoren unter dieser Bezeichnung Verschiedenes verstehen, und unterscheidet daher:

1. Netzbrliche (Epiplocele), in denen sich das stark verfettete Netz befinden kann, oder sogar ein wirkliches Lipom des Netzes.

2. Fettbriche (Hernia lipomatosa), bei denen um den Bruch Fettgewebe sich sammelt und ihn reichlich umwächst.

Fälle, in denen ein Bruch nicht vorbanden ist, sondern in der Richtung desselben sich ein Tumor (Lipom) bildet. Derartige Fälle bezeichnet man als Lipoma herniosum. (Bei dieser Gelegenheit erwähnt V. einen Fall von einem uberaus grossen Lipom, das $191 / 2$ Pfund wog und von Wilms operirt wurde.) Diese Geschwülste bilden sich am häufigsten im Nabel, im Leistenkanal und im Samenstrange, seltener im Annulus cruralis u. s. w.

In demselben Abschnitte uber Lipome spricht Virchow uber das Lipoma scroti, das aus der Tunica dartos hervorgeht, also aus einem Gewebe, in welchem normal kein Fettgewebe zu finden ist. Er erklärt dies damit, dass dieses Gewebe der Ueberrest einer früheren subcutanen Schleimgewebes ist, also die Stelle des Fettgewebes einnimmt, und auch desbalb später ausnabmsweise Lipome bilden kann. Darauf komme ich noch zu sprechen, wenn ich die Aetiologie 
der Lipome behandeln werde. Ich führe. diese Geschwülste nur deshalb an, weil sie, trotzdem sie sehr selten vorkommen, bei der Diagnose zu Verwechseluugen Anlass geben können; man muss sie auch desbalb berticksichtigen, weil sie zu derselben Gattung gehören.

Auch $\mathrm{K}$ ocher ${ }^{1}$ ) erwähnt die Lipome, die aus dem Unterbautzellgewebe des Bauches (Lipoma seroti) bervorgehen. Diese Lipome sollen gewöhnlich mit dem samenstrange im Zusammenhang stehen und werden riesengross, obwohl der Verfasser kein einziges Beispiel dafür anfubrt. Erst in dem Abschnitte uber Lipome des Samenstranges ${ }^{2}$ ) berichtet er von einem Fall ron Lipom, das aus dem subcutanen Gewebe des Bauches hervorging, von Gray operirt und von Curling beschrieben wurde.

Kocher behauptet, dass auch die eigentlichen Lipome des Samenstranges, die aus der Tunica vaginalis communis hervorgehen, die fibrösen Stränge der Scheide auseinanderdrängen, bis in das subcutane Gewebe reichen und den subcutanen Lipomen gleichen hönnen. Es ist klar, dass in einem solchen Falle die Diagnose sebr scbwer sein kann. Sarazin ${ }^{3}$ ) ist hierin der gleichen Ansicht. Andererseits können aber subcutane Lipome bis in die Tiefe der Tunica infundibuliformis dringen und wieder Lipome des Samenstranges vortäuschen; dies sind jedoch offenbar uberaus seltene Fälle, da nur ein einziger von Waltherd) bekannt ist, der von sarazin angefubrt wurde.

Schon Malgaignes) spricht in seiner Abhandlung: „De hernies graisseuses", sehr dentlich über Lipome des Sameustranges, obwohl er sie mit einem anderen Namen bezeichnet. Er zählt 3 Arten dieser Lipombriche: die einen liegen im Samenstrange und jeichen bis zum Bauchfell, bis zum subserösen Gewebe, das ihr Ausgangspunkt zu sein scheint; es ist doch wahrscheinlichcr, dass sie sich aus dem im Strange selbst angesammelten Fett entwickelt haben: Peut-être eût-il fallu les decrire comme des tumeurs graisseuses, développées dans le cordon. Elles forment comme elles une masse unique et distincte; enfiu elles ne sont jamais accompagnées d'un prolongement du peritoine". Die anderen entwickeh sich entschieden in der Bauchhöhle selbst und treten gleich Anfangs als Lipombrüche auf. Die 3. Art entwickelt sic'! im Scrotum um einen alten Bruclisack herum.

Brossard ${ }^{6}$ ) unterscheidet 2 Arten von Lipomen: solche, weiche aus dem subserösen Fettgewebe entstehen, und solche, die sich aus den

1) Kranklieiten der männlichen Gescblechtsorgane. Kap. XI.

2) Kocher, l. c. Kap. XXXVII.

3) Coutribution a l'étude des lipomes du cordon spermatique. Thèse de Paris 1s!10. S. 24.

4 Soc. anat. October 1590). S. 430 (s. Sarazill).

5) Sarazin, l, c

6) Bull. Soc. anat. Yaris 1882, p. 432. 
Fettlappen des Samenstranges entwickeln. In dem von ihw beschriebenen Falle wird uns am deutlichsten die Geschichte der Entstehung und der Entwicklung der Neubildung geschildert. Am Anfange des Jahres 1876 bemerkte der Kranke zufälliger Weise an seinem linken Hoden eine Geschwulst von der Grösse einer Haselnuss; allmählich erreichte die kleine Geschwulst die Grösse eines Eies, und gleichzeitig neben ihr fingen an sich neue kleine Geschwülste zu zeigen. Als nun später im Jahre 1882 der Kranke sich zum Zwecke der Operation einfand, war die Geschwulst schon mannskopfgross und fülte nicht nur den Hodensack aus, sondern reichte auch tief in den erweiterten Leistenkanal. 1)

Aehnlicher Ansicht hierüber sind M o no d und ' Terrill o n ${ }^{2}$ ); sie nehmen jedoch nur einen Ausgangspunkt für die reinen Lipome des Samenstranges an, nämlich die Fettlappen des Stranges.

M. Reynier ${ }^{3}$ ) hingegen ist ganz entgegengesetzter Ansicht. Er meint nämlich, dass der gewöhnliche Ausgangspunkt dieser Geschwülste das subperitoneale Fett ist: „et non pas comme le croient MM. Monod et Terrillon, uniquement les quelques amas de graisse, disséminés dans le cordon". Reynier nimmt den Samenstrang selbst als Ausgangspunkt nur für die Tumoren an, die in den Leistenkanal nicht hineindringen. Offenbar ist er im Unrecht, wie wir dies auch z. B. aus dem Fall von Brossard und Rossander ersehen (Sarazin). Der von Kocht) beschriebene Fall spricht ebenfalls deutlich dafür, dass die Neubildung aus dem Samenstrange hervorgegangen ist, sich aber später im Leistenkanal als eine besondere kleine Gesch wulst entwickelt hat, die einem Bruch täuschend ähulich war.

Gerade ungekehrt, also übereinstimmend mit Reynier's Ansicht, verhält es sich in dem Falle von Watson, Gross, Curling und Anderer, wo die Geschwulst sicher aus dem subperitonealen Gewebe hervorgegangen und erst später in den Hodensack beruntergewachsen ist: „a suivi une marche nettement descendente".

Nach Kocher finden sich im Samenstrange schon im normalen Zustande Fettlappen vor, welche die Bedeutung des subserösen Fettes baben, und welche der Ausgangspunkt für Lipome sind.

Um mir hieruber mein eigenes Urtheil zu bilden, untersuchte ich die Verbältnisse an ca. 5o Leichen und kam zu folgenden Resultaten:

In jedem Samenstrange obne Ausnabme (sogar bei sehr mageren Individuen) findet man $z$ wischen dem perivasculären losen Bindegewebe um den Processus raginalis peritonei makroskopisch sichtbare Fettlappen, die ge-

1) So sagt auch der Verfasser: „Ces tumeurs prennent naissance le plus souvent dans le tissu cellulaire de la partie terminale du cordon sp. sous la forme de petites boules molles allongées, bieu distinctes du testicule et de l'epididyme.“

2) Maladies du testicule et de ses anneces I. Edition. p. 732.

3) (s. Francois Hue.) Bull. Soc. chir. 1593.

4) Deutsche Zeitschr. f. Chir. 18y0. Bd. XXXI. 
wöhnlich im ununterbrochenen Zusammenhange mit dem subperitonealen Fettgewebe stehen und in dinnen Streifen längs des Leistenkanals und des Samenstranges bis zu den Nebenhoden sich hinziehen.

In einigen Fällen, namentlich bei wohlbeleibten Individuen, fand ich das Fett in einer grösseren Menge vor. Einmal habe ich sogar einen ziemlich grossen Fettcylınder bemerkt, der als ein dickerer Strang durch den Leistenkanal bindurchging und stellenweise kirschkerngrosse, rosenkranzartige Verdickungen aufwies.

Vielleicht war dies eine gewöhnliche Hypertrophie des Fettgewebes, die der allgemeinen Fettleibigkeit entsprach, vielleicht auch ein beginnendes Lipom, das der Tod des Individuums sich nicht mehr entwickeln liess.

Alle Fettgebilde des Hodensacks im allgemeinsten Sinne dieses Wortes können wir, dem Beispiele Ledderbose's 1) folgend, ana$\log$ seiner Eintbeilung in 6 Gruppen theilen:

1. Netzhernien, die im Leistenbruchsack einen freien oder angewachsenen Theil des Netzes enthalten, das manchmal in eine grosse Fettgeschwulst sich umwandeln kann. (Zur Erläuterung und zum Vergleich fitge ich sechs schematische Zeichnungen hinzu, fünf nach Ledderbose, die sechste nach Koch.)

2. Eigentliche Fetthernien, die man in Fettgeschwillste eingeschlossene Brüche nennen könnte, und die sich uns derartig entstanden vorstellen, dass um den zuerst hervorgetretenen Bruchsack es zu einer grösseren oder kleineren Ausbreitung von subserösen Fettgewebe gekommen ist. Diese Thatsache ist längst bekannt und wurde ausführlich von Cloquet (l. c.), Werner, ${ }^{2}$ ) szokalski, ${ }^{3}$ ) Annandale u. A. bebandelt.

3. Sogenannte bruchähnliche Fettgeschwülste - Wernher entsteben an der Stelle der Leistenbruche. Das Vorbandensein eines Bruchsacks ist jedoch zu ihrer Charakteristik nicht nothwendig; wenn sich aber ein Bruchsack vorfindet, so entstand er erst secundär durch Zug am Bauchfell, das trichterförmig durch das Gewicht des wachsenden Lipoms zu einem Bruchsack ausgezogen wurde (Fig. 3). Wie wie sehen, ist dies ein vorubergehender Zustand, ent-

1) Die chirurgischen Frkrankungen der Bauchdecken u. s. w. Deutsche Zeitschr. f. Chirurgie. 1890. S. $42 \mathrm{ff}$.

2) Virchow's Archiv. 1869. Bd. XLVII. S. 178. 472.

3) Ueber Fettleibigkeit bei Syphilis und über Fettanhäufungen, die Syphilis vortăuschen. Jahresbericht des Warschauer Aerzte-Vereins (Pam. Tow. lek. warsz.). 1557. XXXVIII, S. 125 . 
weder der Anfang eines eigentlichen Fettbruches, wie ihn ungefähr vor einem Jahrhundert $\mathrm{Cl}$ oquet erklärte, oder ein gewöhnliches Lipom des Leistenkanals und des Samenstranges.

4. Präperitoneal entwickelte Lipome innerbalb eines primären Bruchsackes, deren Entstebung von den Autoren (Fle ury, Szokalski in der Weise erklärt wird, dass ein in der Nachbarschaft eines Bruches aus dem subperitonealen Fette entstandenes Lipom gewissermaassen sich in den Bruchsack vorbaucht, der es allmählich umfasst und umbïllt (Fig. 4).

Wird nun ein solcher Brucbsack abgeschntirt, so entsteht eine seröse Tasche, die in sich ganz geschlossen ist und das Lipom des Samenstranges theilweise umbuillt, wie wir es im Falle Koch's sehen. Letzterer jedoch erklärt aul eine andere Weise das Vorhandensein dieses Sackes in seinem Falle (s. Fall 19).

Nebenbei erwähnen wir an dieser Stelle noch eine 5. Art, näm. lich die subcutanen Lipome des Hodensacks, über die wir ganz ausfubrlich bei der Besprechung der Ansichten Vir cbow's u. Kocher's berichtet haben.

5. Die eigentlichen Lipome des Samenstranges. -- Es ist leicht begreiflich, dass die Diaguose dieser Geschwillste während der Operation selbst und sogar auf dem Secirtisch manehmal nicht geringe Schwierigkeiten bereiten kann; was soll man erst von der klinischen Diagnose sagen. Andererseits unterliegt es keinem $Z$ weifel, dass alle diese einzelnen Arten sich mit einander verbinden, ja sogar die einen in die anderen ubergehen und daher oft grosse Täuschungen bei der Diagnose verursachen können.

Hieraus folgt aber nicht, dass die theoretische Einleitung keinen bervorragenden wissenschaftlichen Werth haben und auch von keinem praktischen Nutzen sein sollte.

Was nun die eigentlichen Lipome des Samenstranges anbetrifft, so theilen wir sie auf Grund ihrer Pathogenese in 2 Gruppen ein:

a) Primäre Lipome des Samenstranges, d. h. solche, die hervorgegangen sind aus einem oder mebreren Nestern des localen Fettgewebes, das zwischen der äusseren Oeffinung des Leistenkanals und dem Hoden ausgebreitet ist.

Diese Geschwülste können wäbrend ibres Wachsthums:

1. sich auf den Samenstrang selbst beschränken, obne seine Grenze weder nach oben, noch nach unten zu uberschreiten; oder können, besonders wenn sie grössere Ausdehnung erreichen

2. die sie umgebende Tunica vaginalis commun. durchbrechen, bis unter die Haut weiter wachsen, als Lipome des Hodensacks 
(Gray $\left.{ }^{1}\right)$ ) den Hoden und Nebenhoden umgeben, „und sogar die Tunica vaginalis testis propria durchwachsen und so intravaginal werden (K ocher)". Endlich können sie

3 . in den Leistenkanal eintreten und entweder in ihm endigen, oder bis zum Bauchfell durchgehen und dort sich gewissermaassen im subserösen Gewebe verbreiten.

b) Secundäre Lipome des Samenstranges, also solche, welche ihren Anfang in der Nachbarschaft des Samenstranges nehmen und später erst wachsend seinen Platz einnehmen. Diese wiederum können entstehen :

1. aus dem Unterbautzellgewebe des Bauches und des Hodensacks (Walther $\left.{ }^{2}\right)$ );

2. von der Aussenseite der Tunica vaginalis communis und des M. cremaster. Letzteres soll sehr selten vorkommen und nicht sicher feststehen (Park, Monod und Terrillon $\left.{ }^{3}\right)$ ).

3. Endlich aus dem subserösen Fettgewebe, also subperitoneal. Von hier steigen sie herunter längs des Leistenkanales und umgeben den Samenstrang. Diese letztere Art von Lipomen ist kein eigentliches Lipom des Samenstranges, sondern ein Fettbruch oder eine bruchähnliche Fettgeschwulst.

Alle Lipome, die in den Leistenkanal sich fortsetzen, sind schwer zu diagnosticiren und können mit verschiedenen Formen von Brüchen verwechselt werden und werden oft genug als solche Jahre lang behandelt (Curling u. s. w.).

\section{Bau, Gestalt und Grösse.}

Lipome des Samenstranges sind aus Fettgewebe zusammengesetzte Geschwilste, und zwar aus der echten "Tela adiposa", ähnlich wie gewöhnliche subcutane Lipome ("Lipomata tuberosa" Virchow). Das Lipom des Samenstranges entsteht aus dem präexistirenden Fettgewebe und, solange es klein ist, kann es der Ausdruck einer gewöhnlichen Hypertrophie der localen Fettzellen und nicht eine Hyperplasie sein, gerade als ob in jeder alten Zelle eine Zunahme von Fett eingetreten wäre. (Excrescentia membranae adiposae - Morgagn $\left.\mathrm{i}^{4}\right)$ ). Aehnlich, wie das Fibrom zur Elephantiasis, verhält sich das Lipom zur Polysarcie.

1) Sarazin, 1. c., Kocher, 1. c., Curling, der den Fall von Gray citirt.

2) Von Sarazin citirt l. c.

3) Sarazin, l. c. sagt so: „mais en tenant compte des observations prises d'une façon rigoureuse, il nous est impossible d'admettre une semblable pathogénie. Loin de nous cependant lidée de la nier."

4) Ganz anders verhălt es sich mit dem Lipom des Hodensacks. Hier enthält, wie ich schon darauf autmerksam machte, die primăre Tunica dartos ganz 
Ein Lipom enthält ein mebr flüssiges Fett, daher ist es weich und zeigt so deutlich eine Fluctuation; freilich aber nur dann, wenn es wenig Bindegewebe entbält. Oft jedoch findet sich das Bindegewebe, das gewöbnlich in geringer Menge die einzelnen Zellen, sowie auch ganze Lappen umgiebt, in grosser Menge vor, so dass dann das Lipom härter erscheint: ist aber sehr viel Bindegewebe vorhanden, so bezeichnet man eine solehe Geschwulst als ein Lipoma fibrosum - Tumeur adipo-fibreuse. Im Samenstrange erscheinen nun diese Neubildungen verschieden hart, wiewohl wir meistens weiche finden. Diese Geschwillste verursachen durch ihre Elasticität und Fluctuation sehr bäufig diagnostische Irrthumer, indem sie Cystengeschwulste des Hodensacks (Hydrocele, Haematocele scroti) vortäuschen. Eine solche Verwechselung wird noch um so mebr dadurch ermöglicht, dass die den Tumor umgebende Hülle gewöhnlich eine verdickte, starke und harte Membran ist. Das Fett, welches diese fest verscblossene häutige Höhle ausfüllt, giebt das Gefuibl einer so deutlichen und ausgesprocbenen Fluctuation, dass es in der That schwer ist, mit der Benennung "Pseudofluctuation“, die sich hier durch gar nichts von einer wirklichen Fluctuation unterscheidet, einverstanden zu sein. Ueberhaupt, glaube ich, wird die Bezeichnung „Pseudofluctuation" etwas zu viel gebraucht, als ob wir z. B. die Fluctuation einer mit Wasser angefüllten Cyste von einer mit Blut oder Oel angefiullten unterseheiden wollten.

In dem Bindegewebe, das kleinere und grössere Lappen umgiebt, zieben sich oft ziemlich zahlreiche Gefässstränge hin, die gewöhnlich an mebreren Stellen, obne einen Hilus zu bilden, in die Neubildung eintreten und sich wie ein Netz in der Geschwulst aus. breiten. Oft treten mit ihnen zusammen Fortsätze der membranösen Hülle der Neubildung ') in die Tiefe hinein.

Die Gestalt und Grösse des Lipoms des Samenstranges ist verschieden, abhängig davon, in welcher Entwicklungsperiode wir es finden; es giebt kleine Geschwülstehen von der Grösse einer Haselnuss oder einer bleifederdicken, kleinen Walze und auch riesengrosse Geschwillste, die mannskopforross oder noch gar umfangreicher werden.

und gar kein Fett, das Lipom ist also ansnahmsweise beteroplastiscb. Dies ist eine gleichsam physiologische Heterologie. „Allein die crassen Humoralpatloologen sind damit nicht zufrieden gewesen, sie halıen für alle Lipome einen dyskrasischen Ursprung angenommen und geradezu von einer lipomatösen Dyskrasie gesprochen (Virchow)."

1) „En resumé, trois éléments prenueut part à la constitution du lipome, la cellule adipeuse, le faisceau conjonctif et l'êlément vasculaire" (Quénu). 
Die Gestalt und die Grösse werden verglichen mit der einer Nuss, eines Apfels, einer Birne, eines Strausseneies, eines Kinds- und eines Mannskopfes, einer Melone u. dg]. Bisweilen werden diese Gebilde riesengross. In dem Falle von Gascoyen war die Geschwulst liber 5 Pfund schwer, in meinem fast 5 Pfund, in dem von Brossard 9 Pfund, von Hue 11 Pfund, von Virchow 191/2 Pfund (Wilms), von Wilson 20 Pfund. Manchmal besteht der Tumor aus mebreren Geschwülsten von verschiedener Grösse und Gestalt, was auf ein Entstehen der Neubildung aus mehreren Herden hinweist.

Der Inhalt der Geschwulst ist zuweilen nicht gleichförmig, wie z. B. in unserem Falle, wo die Mitte stark fibrös und hart war, die peripheren Schichten aber aus reinem Fettgewebe zusammengesetzt, weich und fast zerfliessend waren. Im Falle ron Curling war an der Spitze der Geschwulst eine Cyste und am unteren Pole ein Eiterherd; im Falle von $\mathrm{H}$ ue war die Geschwulst in dem oberen Theile ein reines Lipow, itn unteren colloid entartet; im Falle von Schwart Sarazin fand nan neben dem Lipom des Samenstranges ein Sarcoma des Hodens; endlich beschreibt Duplo a y eine Neubildung des saneustranges, die aus einem Myxosarkom und einem Lipom zusanmengesetzt war. Hier kommen wir schon zu der Cxruppe der gemischten Neubildungen, die ich in der vorliegenden Arbeit zu beschreiben nicht beabsichtigte. Aus diesem Grunde habe ich auch den Fall von Duplouy nicht in der Reihe der sicberen und reinen Fälle untergebracht. Gemischte Neubildungen sind hier nichts Sonderbares.

- Was die Limwandlung eines Lipoms in eine maligne Neubildung betritft (wofür vor Allem Recidive der Geschwulst nach der Operation sprechen, die ron Curling, Langenbeck a. s. w. beschrieben worden sind), ist die Sache noch vicht entschiedeu. „Sans nier la possibilité de cette metamorphose, Broca couclut qu'il n'en existe pas de faits convaincant dans la science" (Reclus).

Das Verhältniss der Neubildung zu der Umgebung ist sehr verschieden, in erster linie ist dies abhängig von der Grösse der Geschwulst.

Die Haut ist immer frei und über der Geschwulst bequem verschiebbar, was sogar cin ziemlich wichtiges Merkmal bei der Diaguose ist.

Kleine Geschwïlstchen, ohne Rücksicht darauf, ob sie aus dem subperitonealen Fett bervorgegangen sind oder aus dem Strange selbst, vereinigen sich uicht eng mit der Umgebung, lassen sich vielmehr sehr leicht von dem umgebenden Gewebe trenuen. Anders 
verbält es sich mit den grossen Lipomen. Diese ungeheuer grossen Geschwulste, die oft die Scheide des Stranges sprengen und nach aussen zum Vorschein kommen oder in die Tunica vaginalis testis propria hineindringen, umwachsen oft das Vas deferens und die Gefässe und sogar den ganzen Hoden mit dem Nebenhoden in der Weise, dass diese Theile gewissermaassen in dem Körper der Neubildung sich verlieren. Die Lage des Hodens mit Rucksicht auf die Geschwulst kann verschieden sein; in unserem Falle lag der Hoden an der inneren, hinteren und unteren Seite der Geschwulst, ähnlich wie in dem von Curling; im Falle Brossard's war der Hoden etwas verkleinert und befand sich an der Innenseite des Lipoms, in dem von Hutchinson an der Hinterfläche und in dem von Koch am unteren Pol der Geschwulst, ähnlich wie dies bei den Cysten der Nebenhoden (Spermatocele) der Fall ist. Sofern es sich nicht um Ausnahmefälle bandelt, bei denen in der Neubildung Entzlundungsprocesse sich abspielten, lässt sich der Hoden immer mit Leichtigkeit von der Geschwulst trennen.

Anders verbalten sich die Blutgefässe, wie auch das Vas deferens. Oft entwickelt sich die Neubildung in ihrer Nachbarschaft, ohne sich mit ihnen zu vereinigen; manchmal jedoch werden sie von der Geschwulst umwachsen und fest umgeben, so dass von einer Trennung und Auspräparirung keine Rede sein kann. Die Gefässe and das Vas deferens gehen einfach durch die Mitte der Geschwulst bindurch. Mitunter senkt sich das Vas deferens, das unabhängig von der Geschwulst verläuft, erst in dem Leistenkanal in die Masse des Lipoms hinein und wird dort vom Fettgewebe bis zur Mundung begleitet. „Mais un peu au-dessous de l'anneau externe, le canal déférent s'engage au milieu du tissu adipeux et le lipome, au sortir de l'anneau interne, continue son chemin vers le bassin et se prolonge, par une extrémité effilée, autour du canal spermatique, qu'il accompagne jusqu' à l'origine des vaisseaux déférentiels" (B roca).

Der Leistenkanal selbst ist oft erweitert - und durch einen Fortsatz oder einen Stiel der Neubildung ausgefullt, was mit Ruteksicht auf Brüche, welche nach der Operation entstehen können, von Wichtigkeit ist.

Ueber das Verbältniss der Neubildung zum Bauchfell habe ich bei der Besprechung der Pathogenese des Lipoms des Samenstranges ausfübrliche Bemerkungen gemacht; daher gehe ich zu einem weiteren A bschnitt iiber. 


\section{Aetiologie - Häufigkeit.}

„L'êtiologie des lipomes du cordon est fort vague" sagt Sarazin mit Recht, denn die Ursachen der Neubildungen überhaupt sind heutzutage ebenso unbekannt wie frïher, und gerade vom Lipom kann man nur dies sagen, dass von keiner von den gutartigen Neubildungen so viel über Aetiologie geschrieben wurde, wie von diesem.

Es würde weit über den Rahmen dieser Arbeit gehen, wollte man sich uber alle diese verschiedenartigen Vermuthungen und Theorien verbreiten, die mitunter sehr interessant sind. lch fuhre nur die schöne Arbeit von Grosch an, die mich das klinische Material aus den letzten 10 Jahren zu sammeln veranlasste, um, wenn nichts mehr, so doch wenigstens die Häufigkeit der Lipome der verschiedenen Körpertheile und vor allem des Samenstranges zu zeigen.

Das klinische Material der letaten 10 Jahre, das meistentheils ambulatorisch behandelt wurde, stellt sich folgendermaassen dar:

Anzahl der Lipome $49\left\{\begin{array}{l}\text { Männer . . . . . } 22 \\ \text { Frauen . . . . . } 27\end{array}\right.$

Was das Alter der Patienten anbetrifft, so fanden wir:

im 75. Lebensjahre . . . . . 1 Lipom

zwischen $60-70$ Jahre . . . . . $3=$

$50-60=$. . . . $6=$

$40-50=. \cdot . \cdot 20=$

$30-40=$. . . . $9=$

$20-30$

$10-20$

unter 10 Jahren ( 2 a. 3 Jahre alt) . 3

\section{Zusammen 49 Fälle.}

Der Localisation nach wurde, wie allgemein festgestellt ist, das Lipom am häufigsten am Rücken vorgefunden, und zwar

$11 \mathrm{mal}$

sodann an der Schulter und Oberarm . . . $9=$ am Nacken . . . . . . . . . . . . $6=$

an der Brust und Achsel . . . . . . . $6=$

am Gesäss und Oberschenkel . . . . . $6=$

am Halse . . . . . . . . . . . . . $3=$

am Bauche . . . . . . . . . . $2=$

an der Stirn . . . . . . . . . . $2=$

am Vorderarm . . . . . . . . $2=$

an der Zunge . . . . . . . . . . . $1=$

am Samenstrange . . . . . . . . $1=$

Zusammen 49. 
Als ein ätiologisches Moment gab man mehrfach ein Trauma an, und ausserdem beobachtete man $2 \mathrm{mal}$ symmetrisch liegende Lipome.

Das Durchschimmern des Lipoms hat man 3 mal festgestellt.

Also unter 49 Lipomen, die man in die poliklinischen Bucher der Krakauer chirurgischen Klinik eintrug, war nur ein Fall von Lipom des Samenstranges. Dieser Procentsatz ist unzweifelbaft zu hoch im Vergleich zu grossen statistischen Zusammenstellungen, in denen diese Localisation kaum einmal auf tausend Fälle vorkommt, und wenn wir erwägen, wie verbältnissmässig wenig Fälle von $\mathrm{Li}$ pomen des Samenstranges bis jetzt veröffentlicht wurden. Jedenfalls nimmt es auch in unserer Statistik, was die Häufigkeit anbetrifft, die letzte Stelle ein.

Im Allgemeinen muss man annehmen, dass jedes Lipom irgend welche locale Ursache (Reizung - irritamentum) bat, die desto kleiner sein kann, je grösser die allgemeine Neigung ist. Diese Disposition kann entweder angeboren (erblich, ähnlich wie bei der Fettleibigkeit) oder erworben sein infolge Dyskrasie (reichliche, fette Ernährung, Alkoholismus u. dgl.). Jedenfalls muss irgend ein Reiz vorhanden sein, der die Fettzellen nicht nur zur Vermehrung anregt, sondern ibnen auch die Kraft zur fortwäbrenden Erhaltung des Fettes verschafft. Daher könnte man eher dem ganzen Organismus das Fett entziehen (z. B. durch Hungern), als das Lipom zum Schwinden zu bringen (Virchow). Ein Trauma kann eine solche Entstebungsursache eines Lipoms sein (Crruveilhier). Wir kennen sogenannte „Berufslipome", wir wissen, dass der Alkoholismus, bequemes Leben, Erblichkeit, Alter, das weibliche Geschlecht zur Bildung dieser Neubildung disponiren, endlich können wir mit Sicherheit besonders bei symmetrischen oder multiplen Lipomen nervöse oder neurotrophische Einflusse annehmen.

Grosch sagt, dass Lipome am meisten in den Körpertheilen entstehen, wo es wenig Talgdrüsen giebt, und das subcutane Gewebe dunn ist.

Unter den durch mich gesammelten Lipomen des Samenstranges soll nur ein Fall von Hue mit Sicherheit nach einem schweren Trauma entstanden sein (Fall rittlings auf einen Baliken). Da die Frauen hierbei nicht in Betracht kommen, und von der Erblichkeit Niemand Erwähnung thut, so mache ich nur einige statistische Angaben: diese Neubildungen treten am häufigsten zwischen dem 40 . und 60. Lebensjahre auf', im jiingeren Alter sehr selten, in der Kindheit gar nicbt. Im Verhältniss zu den Lipomen anderer Gegenden 
findet man sie sebr selten; jedoch im Verbältniss zu anderen Geschwiulsten des Samenstranges sollen sie noch die häufigsten sein. f Nur in einem Falle Verneuil's (Broca) existirten neben dem Lipom des Samenstranges Lipome am Halse und Rücken. Unter den 30 angefübrten Fällen sind 8 rein anatomische, 22 klinische:

Die bei der Section untersuchten oder in Sammlungen aufbewabrten Fälle sind folgende:

1. Fall von Cloquet (Sectionsprotokoll).

2. $=$ Kocher (Museum Zürich).

$3 .=\quad=$ Curling (Chir. Museum London).

$4 .=\quad=$ Delbet (Sectionsprotokoll).

$5 . \quad=\quad$ Virchow (Museum Berlin).

6., 7. u. 8. Fall von Broca (Sectionsprotokolle).

Die klinischen Fälle betreffen alle ohne Ausnabme Personen, bei denen man die Neubildung durcb eine Operation exstirpirte.

Fälle in denen man das Lipom des Samenstranges diagnosticirte, aber die Diagnose durch die Operation oder Section nicht sicherte, bielt ich nicht fur sicher und habe sie desbalb in der vorliegenden Arbeit nicht beruicksichtigt.

Unter, den 30 veröffentlichten Fällen trat die Neubildung 26 mal einseitig, 4 mal auf beiden Seiten anf.

Auf welcher Seite das Lipom im Hodensack häufiger erscheint, ist schwer zu entscheiden, denn nur in 10 Fällen wurde die Lage genau angegeben. Von diesen 10 lag es 5 mal auf der rechten und 5) mal auf der linken Seite, was der Bebauptung von Hutchinson widersprechen würde, der die linke Seite für die von den Neubildungen bevorzugte hielt und dies ähnlich wie die Varicen des Funiculus sperwaticus ${ }^{1}$ ) erklärte.

\section{Symptome und Verlauf.}

Das Lipom des Samenstranges tritt gewöbnlich unbemerkt auf, Am häufigsten bemerkt der Kranke zufällig, dass sich im Hodensack eine kleine Geschwulst gebildet hat, die Anfangs dem Kranken keine Beschwerden verursacht; mit der Zeit jedoch vergrössert sie sich allmählich und fängt dementsprechend an, ihm binderlich zu sein. Sobald sie aber nach kürzerer oder längerer Zeit eine beträchtliche Grösse erreicht hat, verursacht sie die verschiedenartigsten Beschwerden.

Die Zeit in welcher dieser Vorgang sich abspielt, ist sebr ver-

1) Obwohl in der Zusammenstellung von Sa razin 7 linksseitige und 5 rechtsseitige Fälle sind, sagt er trotzdem: ,Liopinion de $\mathrm{H} u$ tehins o n n'est donc point prouvée. D'ailleurs ce fait n'a aucuse importance": 
schieden. Bisweilen erreicht die Geschwulst in verhältnissmässig kurzer Zeit (nach Koch und Sarazin in 2 Jabren) beträchtliche Dimensionen, bisweilen aber erst nach Ablauf eines Jabrzehntes. Gewöhnlich wächst sie sehr langsam; eine überaus schnelle Vergrösserung der Neubildung muss daber den Verdacht erwecken, dass man im gegebenen Falle mit einer malignen oder gemischten Nenbildung zu thun hat. Die riesengrossen Geschwiilste, die einige Autoren beschreiben, brauchten immer viel Zeit zu ihrer Entwicklung. Das grösste Lipom des Samenstranges, das in der kürzesten Zeit heranwuchs, beschrieb Koch; diese Geschwulst entwickelte sich während zweier Jahren so, dass sie bis zu den Knien reichte, wog jedoch nur 5 Pfund. Das Lipom nnseres Kranken hatte ebenfalls in 2 Jabren fast dasselbe Gewicht erreicht, reichte aber nicht bis zu den Knien. Ein so schnelles Wachsthum der Geschwulst ist jedoch eine Seltenheit. Die ungebeuer grosse Geschwulst von Brossard wog 9 Pfund nach 6 Jahren; die 11 Pfund wiegende Neubildung von Hue brauchte dazu einen Zeitraum von 13 Jahren. Wie viel Zeit die 20 pfundigen Riesentumoren (Wilson's und Wilm's) gebrauchten, bis sie das uberaus hohe Gewicht erreichten, darüber fehlen genaue Angaben.

Jedenfalls verursachen die grösseren Geschwulste den Kranken keineswegs grosse Schmerzen und Bescbwerden, sondern sie werden vielmebr ibnen lästig und unbequem, ein Zustand der mit der Zeit immer misslicher wird. Abgesehen von dem drtickenden Gefubl, ein Krüppel zu sein, und von der sich nicbt verdecken lassenden Entstellung, die jedem fühlenden Menschen Kummer bereitet, erschweren sie sehr das Gehen und hindern oft sogar beim Liegen (Koch). Im Falle von Hue musste der Kranke seine Geschwulst in einem uber dew Nacken befestigten Sacke tragen; beim Sitzen stutzte er sie auf dem Boden, um nicht mit heruntergezogen zu werden. Curling giebt an, dass, so oft sein Kranker sich aufrichtete, die Geschwulst mehr hervortrat und durch das Gefuhl der Schwere lästig wurde. Diese Symptome liessen beim Sitzen und mebr noch beim Liegen nach.

Diese Thatsache erklärt sich durch den erschwerten Blutabfluss aus den erweiterten Venen des Samenstranges, zumal da gewöhnlich der im S. Romanum angestaute Koth diese Symptome verschlimmert. Der Vergleich mit der Varicocele des Samenstranges drängt sich von selbst auf. Häufig wird bei grossen Hodensackgeschwülsten der Coitus unmöglich, da der Penis durch die Geschwulst zur Seite geschoben und fast vollständig verdeckt wird: „la verge est avalée par cette distention des bourses; le coït est devenu impossible" (Broca).

Bisweilen kommt es vor (z. B. in dem Falle von Schwartz und 
Sarazin), dass die Neubildung beträchtliche, zum Theil beständige, zum Theil anfallsweise auftretende Schmerzen verursacht; ausnahnsweise können sngar Symptome äbnlich wie bei einem eingeklemmten Bruch auftreten, was dann die Diagnose sehr erschweren kann.

Bei der Untersuchung finden wir den Hodensack entsprechend der Grösse der Geschwulst vergrössert. Die Haut ist gespannt, leicht verschieblich, oft mit einem Netz erweiterter Venen bedeckt. Das Abtasten und sogar ein ziemlich starkes Drulcken verursacht keinen Schmerz. Die Oberfläche der Geschwulst ist zuweilen uneben, höckrig, gewöhnlicb aber findet man sie glatt, weil die weichen Fettmassen von einer verdickten und sehr gespannten Membran ungeben sind. Diesem Umstande auch verdankt der Tumor eine bisweilen ungemein deutliche Fluctuation, die sich in keiner Weise von der Fluctuation einer wirklichen Flussigkeit unterscheidet. Manchmal kann man, wie z. B. in unserem Falle, beim genauen und starken Abtasten in der Tiefe einen Widerstand herausfüblen, der dem barten, centralen Theil der Geschwulst entspricht.

Die Complication der Neubildung mit einer Hydrocele oder einem Eiterherd oder mit beiden zugleich (Fall von Brossard) kann noch die Ursache anderer besonderer Symptome sein.

Das Lipom des Samenstranges ist nicht durclischeinend und unterscheidet sich dadurch von der Hydrocele des Samenstranges; es läs s t sich jedoch nicht in Abrede stellen, dass es wie sub. cutane Lipome ausnahmsweise durebscheinend sein kann.

In keiner von den oben angefubrten Bescbreibungen wurde dieses Symptom angegeben; nur im Falle von Brossard war die Geschwulst in ibrem unteren Segment durchscheinend infolge einer Hydrocele, welche sich dort befand.

Das Verbältniss der Neubildung zum Vas deferens und zum Hoden haben wir in den anatomisch-pathologischen Bemerkungen beschrieben. Der Hoden liegt gewöbnlich unten und an der inneren und hinteren Seite der Geschwulst, bisweilen ist er ganz von dem Neoplasma ungeben. Trotzdem können wir ihn immer herausfublen oder wenigstens seine Lage durch den eigenthümlichen bein Druck sich einstellenden Schmerz bestimmen.

Bei der Percussion ist uberall über der Geschwulst eine absolute Dämpfung vorbanden. In den Anfangsstadien lassen sich zuweilen kleine Lipome, die aus dem subperitonealen Fettgewebe hervorgeben, ebenso wie ein freier Bruch reponiren. Die Leistendriusen sind selbstredend nie afficirt, d. h. weder angeschwollen, noch schmerzhaft; trotz- 
dem, wie aus vielen Berichten herrorgeht, darauf hin specielle Untersuchungen gemacht wurden. -

Allgemeine Symptome treten nie auf, die Kranken sehen gewöhnlich vorzüglich aus; nur in dem Fall von $\mathrm{Hue}$ zeigte sich in den letzten Jahren eine gewisse Cachexie, die die Diagnose einer malignen Neubildung wahrscheinlich machte; nach der Operation jedoch bat sich der allgemeine Zustand gebessert.

\section{Diagnose.}

Die Diagnose des Lipows des Samenstranges ist oftmals sebr schwer, mitunter unmöglich. Bedeutende Chirurgen waren schon oft im Zweifel, womit sie es eigentlich zu thun hatten: Es kam vor, dass sie eine ganz falsche Diagnose aufstellten. So untersuchten im Falle Curling's im Laufe ganzer Jahre gleichzeitig oder einzeln viele bedeutende Cbirurgen (wie Hab, Thompson, Lawrence, Brodie, Harding, Edwards) und diagnosticirten entweder einen Bruch oder konnten die Natur der Geschwulst iiberbaupt nicht bezeichnen.

Gross sclwankte mit der Diagnose zwischen Netzbruch, Cystosarcoma und Teratoma; Reclus glaubte, ein Lipom des myxomatös degenerirten Hodens vor sich zu haben; $\mathrm{Hue}$ hoffte, ein Sarcom zu finden; Anderson vermuthete Aufangs einen gewöhnlichen Bruch und dann einen Netzbruch; Schwartz uahm ein Gumma des Hodens an.

Karewski, dessen Aufsatz ich nicht anfübre, da die letzte Diagnose in seinem Falle: Lipoma fibro-sarcomatosum lautete, diagnosticirte mit Sicherheit, in Anbetracht des jugendlichen Alters des Patienten und des gleichzeitigen Vorhandenseins einer Hasensebarte, eines Teratoma testiculi. Und wieviel Male haben die Autoren keine Diagnose aufgestellt? Mau muss zugeben, dass die Symptome des Lipoms des Samenstranges fast vollständig die gleichen sind, wie die Synoptome der Neubildungen des Samenstranges im Allgemeinen; handelt es sich aber sicher um eine feste Geschwulst, so werden wir in diesem engeren Kreise den Tumor als ein Lipow leicht bestimmen können. Nicht selten, besonders beim ersten oberflächlichen Untersucben, drängt sich die Vermuthung auf, dass es eine Cyste des Samenstranges und nicht ein Lipom ist; der Grund dafür (wie z. B. in unserem Falle) ist wiederum die Fluetuation, und zwar eine so deutliche, dass man sie von der Fluctuation einer Flissigkeit unmöglich unterscheiden kann. Die Verwechselung ist hier sebr leicht, besonders wenn das Lipom (wie wir das im Falle B rossard's seben) mit einer Hydrocele der Scheidenhülle complicirt ist; dann ist näm- 
lich ein Durchschimmern vorhanden, und auch die Probepunction giebt ein positives Ergebniss. Allerdings kann auch ein kleines Lipom wie eine Hydrocele durchschimmern (wie ich schon erwähnte, sahen wir dies bei unseren Lipomen $3 \mathrm{mal}$ ), andererseits aber schimmert eine Hämatocele gar nicht durch. Es erübrigt also zur Feststellung der Diagnose eine gut und genau ausgefübrte Punction (nur darf nach ihr, wie im Falle Brossard's, kein Abscess in der Neubildung hinzutreten) und zugleich ein starkes, tiefes Abtasten, das, wie auch in unserem Falle, oft einen Widerstand in der Tiefe feststellen lässt, was dann die Diagnose einer festen Geschwulst sichert.

Sarazin übertreibt wohl in seiner Abhandlung, die etwas schulmässig abgefasst ist, wenn er schreibt (l. c. S. 39): „Une ponction ne renseigne pas toujours, surtout lorsqu'on tombe sur une cloison; mais il ne faut pas oublier, que la ponction est un moyen quelquefois dangereux, qu'il faut manier, avec la plus grand prudence."

Auch lässt die Punction andere cystenartige Gebilde unterscheiden, die mit dieser oder jener Flüssigkeit gefüllt sind, und auf die ich nicht näher eingehen will (Hydrocele und Haematocele vaginalis, Cystoma scroti, Cystoma epididymis s. Spermatocele, Cystoma testis, Hydrocele congenita s. communicans, Hydrocele diffusa und Haematocele diffusa s. extravaginalis, Chylocele s. Laporocele u. s. w.). Die Tuberculose des Hodens, glaube ich, kann zu einer Verwechselung bei der Diagnose nicht Anlass geben, obgleich Brossard den Vergleich ziemlich streng durchtübrt.

Brossard (1. c.): "L'integrité du testicule resté distinct de la tumeur, permettra d'éliminer la tuberculisation des organes genitaux, qui est généralement bilaterale."

Es ist auffallend, dass verhältnissmässig so oft ein Lipom mit einem Bruch verwechselt wurde. In Anbetracht der Vergrösserung der Geschwulst beim Aufstehen, beim Pressen zum Stuhlgang u. dgl. liegt die Annahme eines Varix des Samenstranges sehr nahe, aber diese Varicen zeichnen sich durch ibre Weichheit aus, geben nach und fallen beim Druck zusammen.

Dagegen ist dadurch, dass der Tumor in den Leistenkanal sich zuriickziehen und zuweilen Einklemmungserscheinungen darbieten kann, die Verwechselung mit einem Bruch sehr leicht möglich, wie dies die gar nicht seltenen Febldiagnosen zur Genüge beweisen.

Desto leichter kann man sich irren beim Netzbruch, der mit dem Samenstrange verwachsen, also nicht reponirbar ist, und ferner bei einem Fettbruch. Gewöhnlich jedoch ist bei einem Netzbruch 
der Anfang rapider, und der Verlauf abweichend. Das Lipom entsteht unbemerkt, vergrössert sich langsam, aber ununterbrochen; der Netzbruch dagegen tritt plötzlich auf, verändert sich aber nicht, wenn nicht in einem geeigneten Augenblicke neue Theile des Netzes hervorkommen und die Geschwulst plötzlich vergrössern. Ueberdies dehnt der Netzbruch den Leistenkanal energischer aus, und zwar so, dass der Stiel der Geschwulst gewöhnlich breiter ist, als der untere freie Theil des Netzbruches; selbst ein sehr grosses Lipom hat aber, wenn uberhanpt, immer einen dunnen Stiel, der dann in das subperitoneale Gewebe ubergeht. Ausserdem fühlt sich der Netzbruch ganz anders an, ist gewöhnlich hart, fluctuirt nicht und vereinigt sich nicht so eng mit den Bestandtheilen des Samenstranges.

Die „bruchähnlichen Fettgeschwtilste" Wernher's kann man nur bei der Section diagnosticiren, wenn sie uiberbaupt einen besonderen, selbstständigen Platz in der Reibe der Fettgesebwitlste dieser Gegend einzunehmen verdienen.

Von den aus dem Integument des Hodensacks ausgehenden Neubildungen zeichnet sich die Elephantiasis des Scrotums durch so charakteristische Merkmale aus, dass eine Verwechselung überhaupt unmöglich sein dürfte. Das Lipom des Hodensacks entwickelt sich oberfläcblich im engen Zusammenhange mit der Haut, die beim Lipom des Samenstranges vollständig frei ist. Uebrigens ist der einzige bekannte Fall Walther's ein wahres Curiosum, mit dem man in der täglichen Praxis gar nicht rechnen kann. Endlich wird die Differentialdiagnose zwischen einem Lipom und anderen Neubildungen des Samenstranges für benigne Tumoren eine andere sein, wie für maligne. Maligne Neubildungen sind gewöhulich secundär, entwickeln sicb schnell, ergreifen früh Nachbargewebe und die Leistendrüsen, endlich bilden sie Metastasen und verursachen gewöhnlich Localschmerzen und Kachexie.

Unter den gutartigen Neubildungen kommt noch am häufigsten das Lipom und das Fibrom, von dem einige Fälle beschrieben wurden, vor (Jobert-Verneuil, Dubois, Curling, Cooper, Fergusson). Letzteres zeichnet sich durch seine Härte aus, ähnlich wie das Chondroma, das noch eine grössere Seltenheit ist (Tillmanns, Kerr). Das Myom (Trèlat) ist etwas ganz Ungewöhnliches; es geht vom Schwanz des Nebenhodens aus. Das Myxom ist mehr ausgebreitet, schwach abgrenzbar, sehr weich und zittert beim Berubren wie Gallerte. 


\section{Die Prognose}

ist beim sonst normalen Allgemeinbefinden, wie bei allen gutartigen Neubildungen, gut. Wenn auch das Lipom des Samenstranges weder den Tod, noch sonst irgend welche unmittelbare Gefahren herbeiführt, so kann es doch gewisse Complicationen und damit auch schwere Erscheinungen verursachen, die man nicht gering schätzen darf, um so mehr, als die Therapie nicht immer leicht und frei von Gefabr ist. Das stete Wachsen der Geschwulst, die dadurch immer schlimmere und schwerere Symptome nach sich zieht, kann für den Kranken nicht gleichgültig sein. Ferner ist die Umwandlung der anfangs gutartigen Geschwulst in eine bösartige immer möglich, was ubrigens von vorn herein niemals sicher ausgeschlossen ist, so dass selbst eine kleine Geschwulst, wenn sie dem Kranken auch keine Schmerzen verursacht, exstirpirt werden muss. Oft bilden sich auf grossen Geschwülsten infolge übermässiger Spannung der Haut Decubitusgeschwüre; ähnliche Geschwüre können auch auf den Lipomen des Samenstranges entstehen, obwohl in den angeführten Fällen nichts derartiges verzeichnet ist. Endlich können Lipome, die sich in den Leistenkanal vertiefen, die Ursache eines Leistenbrucbes sein. Dies betrifft noch in höherem Maasse diejenigen Lipome, welche aus dem subserösen Gewebe hervorgegangen sind, und die oft fest mit dem Bauchfell verbunden sind und durch Zag nach unten eine trichterförmige Einstülpung dieser Membran verursachen. Es ist jedoch hier nicht möglich, die Theorien darzulegen, die die Aetiologie der Brüche im Allgemeinen ausschliesslich auf diese Thatsache stützen (Cloquet, Roser, Wernber, Berger, Monod und Delagénière und in der letzten Zeit Lueas Championnièrel)).

\section{Behandlung.}

Die einzige Art der Behandlung der Lipome des Samenstranges ist die Enucleation, die man mit Rücksicht auf den unsicheren Charakter, die möglichen Umwandlungen und das beständige Wachsen der Geschwulst stets möglichst friih ausführen sollte. Dies habe ich schon bei der Besprechung der Prognose erwähnt. Uebrigens je kleiner die Gesehwulst, desto leichter die Operation, die Verwachsungen sind geringer $\mathfrak{u}$. s. w. Die Enucleation wird auf folgende Weise ausgeführt: Man führt unter Anwendung aller Cautelen der

1) Le role de la grasse dans les hernies. Journal de la Med. et de Chir. pratique. Paris 1896. 
Aseptik auf der vorderen äusseren Seite der Geschwulst einen Schnitt, der linear oder spindelförmig verläuft, und der von der äusseren Oeffnung des Leistenkanals bis zum unteren Pole der Neubildung sich binzieht. Nach Durchschneidung der Haut und Unterbindung der blutenden Gefässe öffnet man die gewöhnlich verdickte, membranartige Umbilllung der Geschwulst, wonach die charakteristischen Fettlappen hervorquellen. Sodann lässt sich gewöhnlich die Geschwulst und deren kuppelförmiger Fortsatz im Leistenkanal mit Leichtigkeit stumpf ganz ausschälen. Nur die Verbindung mit dem Vas deferens ist bisweilen so innig, dass man den Hoden opfern muss, was jedoch nur höchst ausnahmsweise durchaus nöthig sein düfte. Dies betrifft vorwiegend complicirte Geschwiilste, wo auch aus anderen Rucksickten die Castration nothwendig ist. Obwohl die Fälle, in denen man die Enucleation vornahm, bauptsächlich ältere Individuen betrafen, so muss man doch die Enucleation als Ultimum refugium ansehen, als ein Mittel, das man uur in der Noth anwendet. Bei dieser Gelegenheit mache ich darauf aufmerksam, dass sogar in einem solchen Falle, wo man das Vas deferens mit Leichtigkeit isoliren konnte, die feinen Blutadern oft Schwierigkeiten bereiten, da sie leicht reissen und sebr sorgfältiger und genauer Unterbindung bedurfen. Rücksichtslose Unterbindung kann nicht nur Atrophie, sondern auch Gangrän des Hodens herbeiführen; ungenaue Unterbindung wiederum verursacht oft Nachblutung und Bildung eines Hämatoms, das die Heilung verwickelter macht und sie dadurch verzögert.

In meinem Falle war die Isolirung der Venen sehr schwer; es folgte aucb eine unangenehme Complication, ein Hämatom des Hodensacks, das trotz ausgiebiger Entleerung der Coagula die Krankheit sehr in die Länge zog, zu einer allerdings unbedeutenden Atrophie des Hodens Veranlassung gab und dem Kranken ein dauerndes Andenken in der Gestalt eines kleinen harten Knoteos liess.

Andere post operative Complicationen, die verschiedene Autoren anfubren, und die mitunter den Tod herbeiführten, hängen vor Allem vom Arzte selbst $a b$, seiner „Uebung und Gewissenhaftigkeit, und sollten heutzutage tiberhaupt nicht vorkommen.

Wenn Kocher vor einer Septikämie oder Tetanus warnt (Tizzoni), so thut er dies wahrscheinlich unter dem Einfluss von Erinnerungen aus der vorantiseptischen Zeit.

Was die Recidive nach der Operation betrifft, so handelte es sich in den Fällen Carling's, Langenbeck's und Ander'son's 
wahrscheinlich nicht um reine Lipome, sondern um complicirte Nenbildungen.

Nach Entfernung der Geschwulst muss man in erster Reihe an den Leistenkanal denken, falls er durch einen Fortsatz des Lipoms erweitert war. Denn in einem solchen Falle ist ein offenes Thor für einen Bruch gescbaffen. Man muss daber den Kanal eröffuen, das Lipom vollständig herausschälen, die trichterförmige Ausstulpung (wenn sie vorhanden) unterbinden, schliesslich den Kanal und die Oeffnung zunähen in der Weise, wie manesbei einer Radicaloperation des Bruches thut.

Es würde sich also am meisten die Operation nach BassiniPostempski empfehlen. Entsprechend der Grösse der Geschwulst, dem Allgemeinzustande des Kranken, wie auch der Gepflogenheit des Operateurs führt man die Operation in Narkose oder mit Localanästhesie mit Cocain, Eucain oder nach Schleich aus. Die Localanästhesie ist in dieser Gegend leicht und sicher.

Die Wunde kann man beim aseptischen Verfahren und sorg. fältiger Unterbindung der Gefässe obne Gefahr gänzlick zunähen, nachher muss man aber einen leichten Druckverband anlegen.

\section{Anthang.}

Als ich meine Arbeit schon beendet habe, erschien in der Wiener klin. Wochenschrift eine Mittheilung von R. Porges, die denselben Gegenstand bespricht.

In seinem Falle handelte es sich um eine Mischgeschwulst, einen teratoiden Tumor, der aus einem Fibro-myo-sarkom und Lipom zasammengesetzt war und Verkalkungen zeigte.

Das Zusammentreffen eines Lipoms mit einem Sarkom und Teratom ist ein seltenes, aber nicht „in der Literatur noch nirgends beschriebenes Vorkommniss", wie es Porges behauptet.

Wir kennen die Fälle von Dyplouy, Karewski, Hue, Scbwartz, Sarazin u. s. w., wo das Vorkommniss genau beschrieben wird.

Auch die Untersuchungen Hochenegg's, der „bei 232 genau nntersuebten Hoden nur 3 mal Fettablagerung am Samenstrang in geringer Menge nachgewiesen hat", stimmen auffallend nicht mit meinen Befunden überein, da ich - wie ich das oben schon bemerkt habe - in jedem Samenstrange der 50 untersuchten Leichen makroskopisch sichtbare Fettlappen gefunden habe. 


\section{Literatur.}

1. Albert, E., Lehrbuch der Chirurgie. 1891. Bd. III. S. 596.

2. Anderson, William, The Lancet 14. Octobre 1893. (Sarazin).

3. Annandale, Brit. med. Journ. Vol. I. 1868. (S.)

4. Broca, A., Bull. Société anatomique. 1888. p. 974. (S.)

5. Brossard, a) Lipome du cordon. Bull. Soc. anat. 1832. p. 432 . - b) Des tumeurs solides du cordon. sp. Arch. gener. de médicine. Paris 1884. II. p. 267. (S.)

6. Bryand, The Lancet, 1875. t. II. p. 128. (S.)

7. Buck Gordon, New-York med. Record. 1871. 1. Nov. (S.)

8. Championnière Lucas, Le role de la grasse dans les hernies. Journ. de la Med. et de Chir. pratique. Paris 1896.

9. Cloquet, Recherches sur les causes et l'anatomie des hernies abdominales. Thèse de Paris. $181 \%$.

10. Cur ling, Récidive de tumeurs lipomateuses du c. sp. et du scrotum. Transact. of the pathol. Soc. London XVIII. 1868.

11. Derselbe, Maladies du testicule (Gosselin).

12. Deguise, Bulletin et mémoires de Soc. chir. Bd. IX. 1859. (S.)

13. Delbet, Pierre, Bull. Soc. anat. 1885. p. 974. (S.)

14. Duplay et Reclus, Traité de chirurgie. Bd. VIII. p. 235.

15. Duplay et Follin, Pathologie externe. Bd. VII. p. 379. (S.)

16. Duplouy, Lipo-myzo-sarcoma funiculi. Sem. med. 1891. p. 400.

17. Englis ch, Oest. med. Jahrbücher. 1885. Heft 3.

18. Gascojen, Transact. of the pathol. Soc. London XVIII. 1877. p. 76. (S.)

19. Gross, Revue médicale de l'Est. 1890. p. 184. (S.)

20. Holme s, Lipoma scroti. System of. Surgery. 1876. (4.)

21. Hodges, R. M., Exceptional fatty tumors. Boston 1884. (Centralbl. f. Chir. 1885. Nr. 12.)

22. Hue, Fr. (Rouen), Sem. méd. 1893, p. 541.

23. Derselbe, Bull. Soc. chir. 1893. p. 631. rapport p. M. Paul Reynier.

24. Hu t chins on, Jonathan, Lipomata in hernial regions. Transact. of. the pathol. Soc. London XL.

25. Derselbe, Centralbl. f. Chir. 1887. Nr. 29.

26. Hyrtl, J., Handb. d. topographischen Anatomie. Bd. I. S. 854. (S.)

27. Icart et Aubert, Lipome du cordon. Comptes rendus de séances de la Soc. d. Sciences med. Lyon 1874. (S.)

28. Ko ch, Carl, Beitrag zur Kenntniss der Fettgeschwülste im Hodensack. Deutsch. Zeitschr. für Chir. 1890. Bd XXXI.

29. K o ch er, Theodor, Die Krankheiten d. männlich. Geschlechtsorgane. Deutsche Chir. S. 24. i 184.

30. Ledderhose, G., Die chirurgischen Erkrankungen der Bauchdecken etc. 1890. Deut. Chir. S. 45.

31. Malgaigne, Tumeurs du cordon sp. Th. de conc. pour le prof. Paris 1848. (S.)

32. M o nod et Terrillon, Maladies du testicule et des ses annexes. Ed. I. p. 732.

33. Park, R., Lipoma testis etc. Annales of Surgery. 1886. p. 365. Ref. Centralbl.

f. Chir. 1886. Nr. 38. (Centralbl. f. Chir. 1888! bis).

34. Parker, W., Med. gaz. New-York 1882. Bd. IX. p. 373. (S.)

35. Pean, Tumeurs de l'abdomen et du bassin. T. II. 1885. p. 350. 
36. Reclus, Soc. de Chirurgie. 1888. p. 634 .

37. Ros s ander, Hygiaea 1883. Jahresber. Virchow. 1884.

38. Sarazin, Gaston, Contribution à l'étude des lipomes du cordon Sperm. Thèse de París. 1895.

39. Schwartz, Mal. des organes genit. de l'homme. Encycl. int. de Chir. VII. p. 561.

40. Virchow, Die krankhaften Geschwülste. Bd. I. (Lipoma-Wilms).

41. Waldeyer, Arch. f. pathol. Anat. 1868. Bd. XLIV. Heft 1.

42. Walther, Soc. anat. 1890. p. 430. (S.)

43. Wats on, Eben, The Lancet. London 1885. I. p. 792 i 837.

44. Willemer, (vide Hodges) Referent Centralbl. f. Chir. 1885. Nr. 12.

45. Szok alski, 0 kiłach tłuszczowych i nagromadzeniach tłuszczu našladujacych kiły. - Pamietnik Tow. lek. warszaw. 1857. str. 125. 